Original Research Articles

\title{
Correlation between Non-Alcoholic Fatty Liver Disease (NAFLD) fibrosis score (NFS) with Left Ventricular Mass Index (LVMI) in patients with NAFLD
}

\author{
Tri Ferry Rachmatullah ${ }^{1}$, Cecilia Oktaria Permatadewi ${ }^{2}$, Hesti Triwahyu Hutami ${ }^{2}$, Charles Limantoro ${ }^{3}$, \\ Hery Djagat Purnomo** \\ ${ }^{1}$ Faculty of Medicine Universitas Diponegoro/dr Kariadi Hospital, Semarang, Indonesia \\ ${ }^{2}$ Division of Gastroenterohepatology, Department of Internal Medicine, Faculty of Medicine Universitas \\ Diponegoro /dr Kariadi Hospital, Semarang, Indonesia \\ ${ }^{3}$ Division of Cardiology, Department of Internal Medicine, Faculty of Medicine Universitas Diponegoro /dr \\ Kariadi Hospital, Semarang, Indonesia
}

\section{Article Info Abstract}

History

Received: 01 July 2021

Accepted: 29 Aug 2021

Available: 31 Aug 2021
Background: Cardiovascular-related mortality is a major concern in NAFLD. Advanced fibrosis was known to be associated with cardiovascular diseases. NAFLD Fibrosis Score (NFS) is used to identify the development of liver fibrosis in NAFLD patients. Left ventricular mass index (LVMI) is a sign of subclinical cardiovascular complications in NAFLD. The correlation between NAFLD fibrosis score with LVMI in NAFLD patients is not fully established.

Objective: To analyze the correlation between NAFLD fibrosis score with LVMI in NAFLD patients.

Methods: A cross-sectional study of NAFLD patients in Kariadi Hospital Indonesia. NFS was calculated using a formula based on clinical and biochemical parameters. LVMI was measured with echocardiography. Pearson's, Mann-Whitney, and logistic regression were used for analysis.

Results: A total of 64 patients with primary NAFLD were enrolled, 54.7\% males and $45.3 \%$ females. Mean age was $52.8 \pm 10.5$ years (30-77 years). Based on NFS criteria, the high probability group was most prevalent (50\%), followed by intermediate probability group $(34.4 \%)$ and low probability group $(15.6 \%)$. Highest increase in LVMI was obtained in the high probability group (93.8\%), followed by intermediate probability $(59.1 \%)$, and low probability group (10\%) respectively. There was significant correlation between NFS and LVMI (P 0.002). Logistic regression showed that NFS has a more significant correlation with LVMI compared to gender $(\mathrm{P}=0.002)$. Conclusion: NFS is a non-invasive liver fibrosis score which independently correlates with Left ventricular mass index (LVMI), a marker of cardiovascular abnormality.

Keywords:

NAFLD; NFS; LVMI; NFS with LVMI; NFS with LVMI in NAFLD.

Permalink/ DOI: https://doi.org/10.14710/jbtr.v7i2.11624

\section{INTRODUCTION}

Non-alcoholic fatty liver disease (NAFLD) is currently the main contributor of chronic liver disease and has a variety of clinical spectrum ranging from simple steatosis to non-alcoholic steatohepatitis (NASH).

\footnotetext{
* Corresponding author:

E-mail: herydjagat@yahoo.co.id

(Hery Djagat Purnomo)
}

This condition often leads to fibrosis, cirrhosis, and malignancy. ${ }^{1}$ The prevalence rate of NAFLD in America reached up to $25-30 \%$ of the general population with 2 $-3 \%$ of these patients suffering from NASH. ${ }^{2,3}$ A large prospective cohort study by Stephen et al, found that from middle-age Americans living in Texas, the prevalence of NAFLD was estimated to be at an approximate of $38 \% .{ }^{4}$ Meanwhile, the current prevalence rate of NAFLD in the Asia Pacific was estimated to be 
around $5-30 \% .^{5,6}$ In Jakarta, the prevalence of NAFLD was approximately $30.6 \% .^{7}$ Based on the ultrasonography results obtained from the radiology department, the number of patients with NAFLD in dr. Kariadi hospital Semarang has also increased. ${ }^{8}$

NAFLD has been a 'Western Disease', however in the past two decades, urbanization in many Asian countries has led to a sedentary lifestyle and overnutrition, which is lead to the escalation of obesity and NAFLD cases. ${ }^{9}$ Clinical and epidemiological studies discovered that NAFLD is also correlated with an increased risk of coronary artery diseases, abnormality of heart function and structure (ventricular hypertrophy, ventricular dysfunction, heart failure), valvular heart disease (aortic sclerosis), and arrhythmia (atrial fibrillation). ${ }^{10}$ In a study about NAFLD which followed up the progress of patients for $8-11$ years, death occurred in $13-45 \%$ of these patients. The combination of NAFLD and cardiovascular diseases $(25 \%-28 \%)$ was the highest cause of mortality. ${ }^{2}$

NAFLD has a significant correlation with cardio metabolic risk factors such as obesity, hypertension, dyslipidemia, low insulin-like growth factor-1 (IGF-1), increase of inflammatory factors and hemostasis in plasma, insulin resistance, metabolic syndrome, endothelial dysfunction, abnormal glucose tolerance, and type- 2 diabetes mellitus. ${ }^{10,11}$ Patients with NAFLD especially those at an advanced stage of fibrosis will exacerbate hepatic and systemic insulin resistance. In turn it will induce atherogenic dyslipidemia and release multiple inflammatory factors, pro-coagulant, and profibrinogenic which holds an important role in the pathophysiology of cardiovascular complications. ${ }^{12}$

Liver biopsy is currently accepted as the gold standard for confirming the degree of inflammation and fibrosis in patients with chronic hepatic diseases. However, liver biopsy is invasive, expensive, often produces samples with high variability and has adhering complications. A number of non-invasive methods have been developed by combining clinical and serological variables that are able to determine the occurrence of advanced fibrosis in patients with NAFLD. ${ }^{13}$ NAFLD fibrosis score (NFS) is one of the non-invasive methods that has been validated in its application for determining the degree of liver fibrosis in patients with NAFLD. NFS has been recommended by the American Association for Study of Liver Disease (AASLD), American College of Gastroenterology (ACG), American Gastroenterological Association (AGA) and by the European Association for the Study of the Liver (EASL). ${ }^{13,14}$

NAFLD is known to be a risk factor for the occurrence of subclinical abnormalities in myocardial metabolism and is associated with subclinical changes over time in left ventricular structure function, which causes both structural and functional heart abnormalities. ${ }^{14}$ Left ventricular mass index (LVMI) is an adjusted mass of the left ventricle to the total surface area of the body. This measurement is frequently used as a parameter in examining geometrical changes of the left ventricle. ${ }^{12,15-17}$ A study about the measurement of LVMI using echocardiography M-Mode, showed that patients with NAFLD has a higher value of LVMI than the normal population. ${ }^{18}$
Treeprasertsuk et al. 2013 and Kim et al.2013 revealed that advance fibrosis determined by NFS was a significant predictor for mortality, especially regarding cardiovascular incidents in patients diagnosed with NAFLD through ultrasonography. ${ }^{19,20}$ Studies by Giorgio et al. in 2014 showed that patients with moderate and high value of NFS has a significant correlation with an increase of LVMI. ${ }^{1}$ However, research like this have never been done in Indonesia, especially in Central Java where the incidence of NAFLD is quite high with higher probability of cardiovascular disease. While there are many similarities between patients with NAFLD from Asia and the West, there are several features which may differentiate the patients with NAFLD from Asia, such as BMI, comorbidities, and genetic factors. ${ }^{9}$ Therefore, In this research we will assess the correlation between NAFLD Fibrosis Score (NFS) with Left Ventricular Mass Index (LVMI) of patients with NAFLD in dr Kariadi Hospital Semarang. This study also aimed to look for clinical characteristics of NAFLD in Semarang population compared to Western population.

\section{MATERIALS AND METHODS}

This analytical cross-sectional study was conducted at the Department of Gastroenterology and Hepatology, dr. Kariadi Hospital Semarang between October 2015 and September 2016. Subjects were patients of the Gastroenterology and Hepatology out-patient clinic that fulfilled the inclusion criteria. The inclusion criteria for this study were patients with no history of alcohol consumption or have history of alcohol consumption under 20 grams/day for women and 30 grams/day for men, age $\geq 18$ years old, and was diagnosed with NAFLD by ultrasonography with or without biopsy. The exclusion criteria were hepatitis due to viral infection, alcoholic liver disease, consumption of medicines which may lead to a fatty liver, other liver diseases, arteritis or connective tissue disorders, renal, thyroid, and cardiovascular diseases.

Subjects were collected through consecutive sampling, and the sample size was calculated using correlation coefficient in cross sectional research with minimum sample size was 57 patients. The study was approved by the Ethics Committee of Faculty of Medicine Universitas Diponegoro and dr. Kariadi Hospital number 533/EC/FK-RSDK/2015 and was conducted according to the Declaration of Helsinki. We provided detailed information on the study procedure to the patients, followed by written informed consent from the subjects.

64 patients were enrolled. Interview and physical examinations comprise of vital signs, body weight, height, body mass index, hip circumference, cardiac, and abdominal examinations. Other examination such as echocardiography M-mode, blood (routine blood count, urea, creatinine, lipid profile, uric acid, AST, ALT, albumin, fasting blood glucose, $\mathrm{HBsAg}$, anti $\mathrm{HCV}$, and post prandial blood sugar) and urine examinations were obtained. NAFLD fibrosis score was calculated using an official calculator from www.nafldscore.com. 


\section{RESULTS}

\section{Patient Characteristics}

From the period of October 2015 to September 2016, 64 patients fulfilled the inclusion criteria. The basic characteristics of these 64 patients was presented on Figure 1. 19 patients $(29.7 \%)$ were $\leq 50$ years old and 45 patients $(70.3 \%)$ were $>50$ years old with gender distribution of 29 females (45.3\%) and 35 males $(54.7 \%)$. This study showed that the subjects suffering from NAFLD was dominated by males $54.7 \%$ males vs $45.3 \%$ females) with average age distribution between $55 \pm 10$ years.

There were some comorbidities found on all samples (64 patients) in the gastroenterology and hepatology outpatient clinic in Kariadi Hospital; overweight $(89.1 \%)$ was discovered as the most frequent comorbid followed by dyslipidemia $(81.3 \%)$, increase in abdominal circumference $(70.3 \%)$, increase in ratio of AST/ALT (56.3\%), metabolic syndrome according to IDF 2006 (56.3\%), hypertension (34.4\%), and diabetes (25\%). Ratio of AST/ALT was an interesting discovered in our study. $56.3 \%$ of our subjects have $>1$ ratio which indicates the chronicity of liver disease in patients. Figure 2 shows the comorbidities of these data.

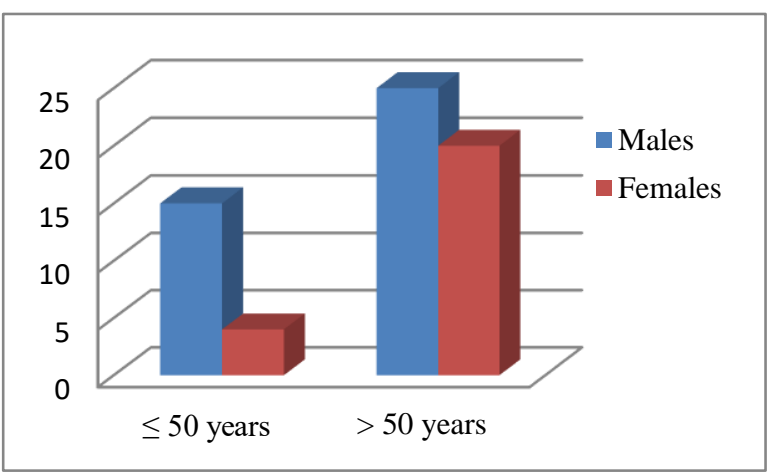

Figure 1. Distribution of subjects according to age and gender

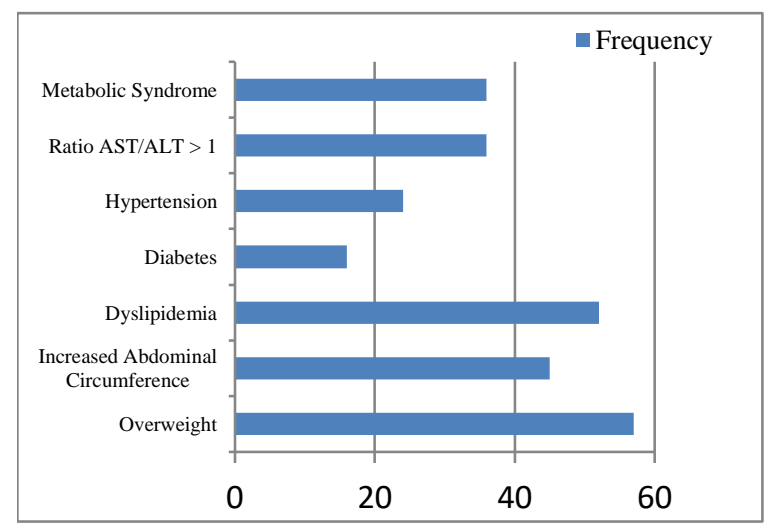

Figure 2. Distribution of Gastroenterohepatological clinic patient's comorbidities

\section{Respondent Distribution based on NFS and LVMI degree}

Subjects were divided into 3 groups in accordance with their NAFLD fibrosis score (NFS) : low probability group consisting of 10 patients $(15.6 \%)$, intermediate probability group consisting of 22 patients (33.4\%), and high probability group consisting 32 patients $(50 \%)$. An increase in LVMI found in 44 patients $(68.7 \%)$. The
LVMI increased in 1 patient in the low probability group, 13 patients in the intermediate probability group, and 30 patients in the high probability group. Figure 3 shows the distribution of these data.

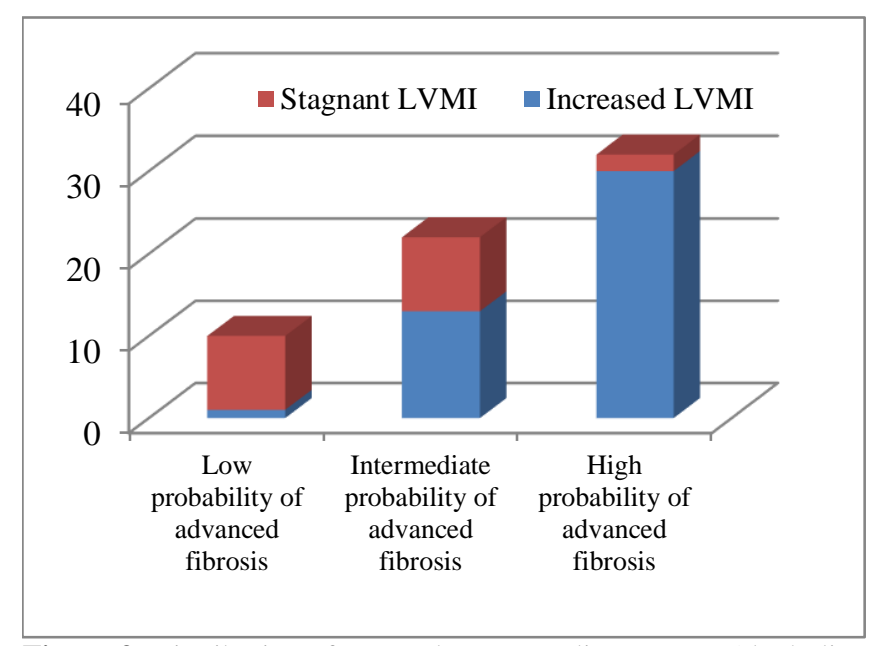

Figure 3. Distribution of respondents according to Non-Alcoholic Fatty Liver Disease (NAFLD) Fibrosis Score (NFS) and Left Ventricular Mass Index (LVMI)

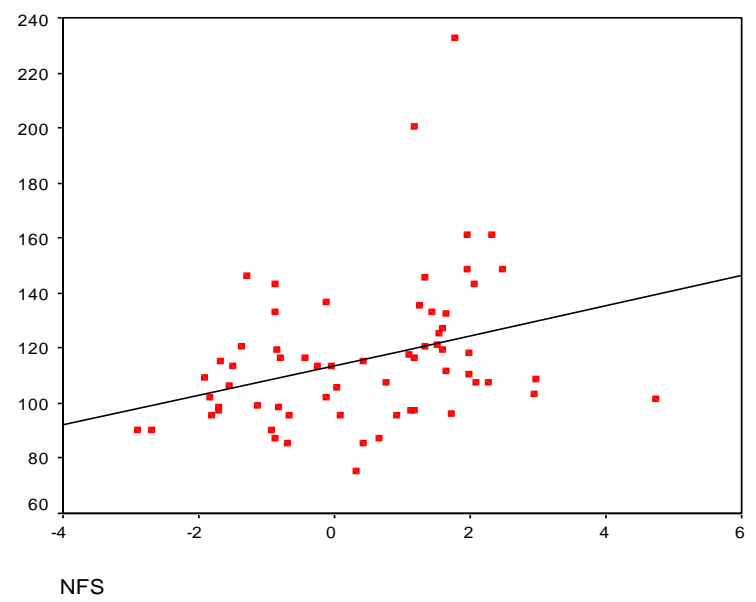

Figure 4. Scatter gram and regression line of Non-Alcoholic Fatty Liver Disease (NAFLD) Fibrosis Score (NFS) in comparison with Left Ventricular Mass Index (LVMI) presenting a linear correlation

\section{Bivariate Analysis \\ Correlation between NFS and LVMI}

Spearman's Correlation analysis showed a positive correlation between NFS and LVMI (P 0.002 r 0.377) as displayed in Table 1. Figure 4 shows a scatter gram representing the correlation between NFS and LVMI. This study discovered that an increase of LVMI occurred in patients with an intermediate or high probability NFS score, thus a higher degree of NFS will cause a more significant increase of LVMI.

Based on bivariate analysis, this study showed that cardiovascular risk profile increases in accordance with NFS score. High probability patients have the worst cardiovascular risk profile. This risk profile gradually decreases in intermediate and low probability patients. 


\section{Confounding Factors Correlation with LVMI}

Comorbidities were categorized as confounding factors that also leads to an increased LVMI. Confounding factors that increased LVMI were gender and hypertension. In this research, Further analysis done by using Mann-Whitney analysis discovered that gender was the only confounding factor that have a significant effect on LVMI $(\mathrm{p}<0.25)$, as displayed in Table 2.

\section{Multivariate Analysis}

Multivariate analysis was done to explore the correlation between dependent and independent variables. Bivariate analysis showed that gender was one of the variables correlated with an increased LVMI. Multivariate analysis using the Enter method showed that NFS was more influential in increasing LVMI than gender ( $P$ 0.002) as showed in Table 3.

Table 1. Positive correlation between Non-Alcoholic Fatty Liver Disease (NAFLD) Fibrosis Score (NFS) and Left Ventricular Mass Index (LVMI)

\begin{tabular}{ccc}
\hline Variable & $\boldsymbol{P}$ & $\boldsymbol{r}$ \\
\hline NFS - LVMI & $\mathbf{0 . 0 0 2}$ & 0.377 \\
\hline * Spearman's Correlation &
\end{tabular}

Table 2. The Correlation between confounding factors with Left Ventricular Mass Index (LVMI)

\begin{tabular}{llcc}
\hline \multicolumn{2}{c}{ Variable } & LVMI & $\boldsymbol{P}$ \\
\cline { 3 - 4 } Gender & Man & \begin{tabular}{c} 
Med \pm SD \\
\multirow{4}{*}{ Hypertension }
\end{tabular} & \\
& Woman & $118 \pm 28$ & $\mathbf{0 . 0 4}^{*}$ \\
& + & $113 \pm 26$ & \\
& - & $109 \pm 27$ & $0.815^{*}$ \\
\hline
\end{tabular}

*Mann-Whitney

Table 3. Multivariate analysis

\begin{tabular}{lccccc}
\hline Variable & \multicolumn{3}{c}{$\boldsymbol{U C}$} & $\boldsymbol{S C}$ & \\
\cline { 2 - 6 } & $\boldsymbol{B}$ & $\boldsymbol{S . E}$ & Beta & $\boldsymbol{t}$ & $\boldsymbol{S i g}$ \\
NFS & 6.647 & 2.022 & 0.395 & 3.287 & $\mathbf{0 . 0 0 2}$ \\
Sex & -14.757 & 6.382 & -0.278 & -2.31 & 0.024 \\
\hline
\end{tabular}

*significant $(\mathrm{p}<0.05)$

\section{DISCUSSION}

From this analytical cross-sectional study of NFS and LVMI in NAFLD patient, it was discovered that the characteristics of the subject were dominated by males. Gender is currently considered as a risk factor of NAFLD. ${ }^{1}$ Incidence rate of NAFLD was higher in $>50$ years old group with $2: 1$ ratio when compared with $\leq 50$ years old group. This is in line with previous study by Lesmana et al. and Lisa et al. who also found that the participants with NAFLD was more likely to be male (54.7\% versus $37.9 \%)$ and were similar in age with a mean of 50.4 years. ${ }^{7,14}$

Overweight was the most common comorbid. The rationale is that in an inflammatory state, fat accumulation in the lower abdomen will stimulate a proinflammatory cytokine release which contributes to the pathophysiology of NAFLD. ${ }^{21,22}$ Purnomo et al. and Adams et al. reported an increased prevalence of NAFLD in accordance with an increased BMI. 23,24 Study by Makker et al. also found that waist circumference, transaminases levels, and left ventricle mass were highest in patients with severe NAFLD and NAFLD with Metabolic syndromes combined. ${ }^{25}$
A study done by Hong et al. showed that the prevalence of dyslipidemia was $66.8 \%$, which was almost similar to our results. It was discovered that hypertriglyceridemia $(80.4 \%)$ and a decrease in HDL $(77.5 \%)$ were the two most common findings. Dyslipidemia is a risk factor and also a contributing factor that exacerbate the progressivity of NAFLD. ${ }^{26}$ Beside fatty acids and triglycerides, evidence showed an increased accumulation of free cholesterol in the liver with subsequent toxic effects contributing to liver damage. ${ }^{10}$ Our study also discovered $34.4 \%$ of subjects suffered from hypertension. It is one of the more influential metabolic syndromes that contributes towards the pathogenesis of NAFLD and a risk factor of cardiovascular abnormality. ${ }^{27}$

Ratio of AST/ALT $>1$ in this study indicated the chronicity of liver disease in patients and reflects the severe progression of NAFLD. ${ }^{28}$ NAFLD typically is characterized by a hepatocellular pattern of liver-related enzymes with mild elevations (1-2 times the upper limit of normal) in serum alanine aminotransferase (ALT) and aspartate aminotransferase (AST) ${ }^{28} \mathrm{AST} / \mathrm{ALT}$ ratio has been proposed to predict progressive fibrosis in NAFLD. Angulo et al, suggested AST/ALT ratio > 1 reflects the severe progression of NAFLD. ${ }^{29}$

Several study have shown that diabetes was one of the main risk factors of NAFLD. This was different compared to our study where diabetes was only found on $25 \%$ of the subjects. ${ }^{26,30,31}$ This difference might be caused by a novel opinion that NAFLD is also a risk factor for diabetes. This opinion is supported by a study of Jonathan et al. that showed $73 \%$ of NAFLD patients developed diabetes later in their life. NAFLD and diabetes can influence one another. Scientific reports by Alexander et al. showed that diabetes was associated with an increased risk of NAFLD and myocardial dysfunction. From this reports, $54 \%$ of NAFLD patients had a NAFLD fibrosis score consistent with advance fibrosis. ${ }^{32}$

In line with previous study, a recent epidemiological studies showed an increase in cardiovascular diseases in patients with NAFLD with no history of traditional risk factors and metabolic syndrome. ${ }^{33}$ This was caused by a complex interaction between NAFLD, insulin resistance, and visceral obesity which leads to an increased risk of cardiovascular diseases in patients with NAFLD. NASH exacerbates hepatic and systemic insulin resistance that causes atherogenic dyslipidemia which is commonly presented with an increase of TG, small dense LDL, and decrease of HDL. NASH also exacerbates the production of pro-inflammatory cytokines (C-reactive protein, interleukin-6, tumor necrosis factor-alpha), pro-oxidant molecules (oxidized LDL, nitro tyrosine, thiobarbituric acid-reacting substances), and pro-fibrinogenic mediators (tumor growth factor-beta, insulin like growth factor-1, endothelin-1). Release of components from the renin-angiotensin-aldosterone system that usually occurs in hypertensive patients was also found in NALFD patients. 1,33

NASH also caused abnormalities in hepatitis m-RNA expression with mediators that are responsible for vascular and cardiac injuries. This strengthens the theory that the increase in factors stated above were caused by hepatic cells that underwent steatosis and inflammation. 6,12 
Experimental studies also discovered that a number of gen (PNPLA3-I148M, MBOAT7, GCKR and HSD17B13), which were involved in the metabolism of fatty acid, lipolysis, coagulation, monocyte and macrophage regulation, and also inflammatory response experienced an overt expression in patients with NASH. ${ }^{12,13}$ Biological factors secreted by the liver also holds a significant role in structural remodeling and geometrical abnormalities of the heart. ${ }^{34}$

Increase of LVMI occurred in patients with an intermediate and high probability NFS score. This result was similar to Giorgio et al. study that discovered the increase of LVMI was not occur in patients with a low probability NFS. A cohort study by Kim et al. found that 241 patients with NAFLD also had an alteration in the geometry of the left ventricle and early diastolic disfunction. The severity of NFS and the increase of LVMI goes in concord. ${ }^{2,35}$

Chun-on et al. found a strong association between NFS with CHD, CHF, and CVD because these scores detect patients with more advanced disease and liver fibrosis. ${ }^{36,37}$ Hokyou et al. found that subject with advanced liver fibrosis exhibited a significantly higher prevalence of left ventricular diastolic dysfunction compared with those without steatosis. ${ }^{38}$ Kuang et al showed that the correlation between NAFLD and cardiovascular diseases are not linked to traditional risk factors of cardiovascular diseases and metabolic syndrome. ${ }^{39}$ NFS contains liver-specific variables, AST and ALT, or together with other shared risk factors, it is suggesting the perspective that liver enzymes AST and ALT may play a significant and independent role in the pathophysiological mechanism mediating the occurrence of the Cardiovascular abnormality. ${ }^{40}$ Therefore, NFS as a non-invasive method for detecting advanced fibrosis could also be used as an indicator to predict the increase of LVMI in NAFLD patients.

However, there were some limitations to this study. Sample collection and NFS calculation were only done once without further follow up even though the progressivity of liver fibrosis could affect calculation. Fibrosis scoring was also done with a single scoring tool without any comparison with other methods. The diagnosis of fibrosis was also not confirmed with histopathological biopsy. It is necessary to conduct this study with conducting subgroup analysis in each group according to the degree of NFS using healthy controls and a prospective cohort study method.

\section{CONCLUSION}

A significant positive correlation was showed between NFS score and LVMI in patients with NAFLD. NFS score might be useful as a predictor for cardiovascular outcome in NAFLD patients.

\section{ACKNOWLEDGMENTS}

Thank you to dr. Galih Prakasa Adhyatma and dr. Herna Rizkia Armatussolikha as research assistant and all the medical staff of the Outpatient Clinic of Internal Medicine at RSUP Dr. Kariadi Semarang who helped with this study and its publishing process.

\section{REFERENCES}

1. Sesti G, Sciacqua A, Fiorentino TV, Perticone M, Succurro E, Perticone F. Association between noninvasive fibrosis markers and cardio-vascular organ damage among adults with hepatic steatosis. PLoS One. 2014;9(8):e104941.

2. Azzam H, Malnick S. Non-alcoholic fatty liver disease - The heart of the matter. World J Hepatol. 2015;7(10):1369-76.

3. Ni L, Yu D, Wu T, Jin F. Gender-specific association between non-alcoholic fatty liver disease and type 2 diabetes mellitus among a middle-aged and elderly Chinese population: An observational study. Medicine (Baltimore). 2021;100(6):e24743.

4. Harrison SA, Gawrieh S, Roberts K, Fournier C, Paredes AH, Alkhouri N. Prospective evaluation of the prevalence of non-alcoholic fatty liver disease and steatohepatitis in a large middle-aged US cohort. J Hepatol [Internet]. 2021;75(2):284-91. Available from: https://doi.org/10.1016/j.jhep.2021.02.034

5. Chalasani N, Younossi Z LJ. The Diagnosis and Management of Non-alcoholic Fatty Liver Disease : Practice Guideline by the American Association for the Study of Liver Diseases, American College of Gastroenterology, and the American Gastroenterological. Am J Gastroenterol. 2012;55:2005-23.

6. López-Pastor AR, Infante-Menéndez J, Escribano Ó. miRNA Dysregulation in the Development of NonAlcoholic Fatty Liver Disease and the Related Disorders Type 2 Diabetes Mellitus and Cardiovascular Disease. Front Med. 2020;7:527059.

7. Hasan I LL. Prevalence and Risk Factors for Nonalcoholic Fatty Liver in Indonesia. Pertem Ilm Tah XIV PPHI. 2007;

8. Sasdesi L. Data Kunjungan Pemeriksaan Ultrasonografi Abdomen Instalasi Radiologi RSUP Dr Kariadi Semarang. 2010.

9. Fan JG, Kim SU, Wong VWS. New trends on obesity and NAFLD in Asia. J Hepatol [Internet]. 2017;67(4):862-73. Available from: http://dx.doi.org/10.1016/j.jhep.2017.06.003

10. Malhotra P, Gill RK, Saksena S, Alferai WA. Disturbances in Cholesterol Homeostasis and Nonalcoholic Fatty Liver Diseases. Front Med. 2020;7:467.

11. Sesti G, Fiorentino T V., Hribal ML, Sciacqua A, Perticone F. Association of hepatic insulin resistance indexes to nonalcoholic fatty liver disease and related biomarkers. Nutr Metab Cardiovasc Dis. 2013;23(12):1182-7.

12. Ballestri S, Lonardo A, Bonapace S, Byrne CD, Loria P, Targher G. Risk of cardiovascular, cardiac and arrhythmic complications in patients with nonalcoholic fatty liver disease. World J Gastroenterol. 2014;20(7):1724-45.

13. Martin K, Hatab A, Athwal VS, Jokl E, Hanley KP. Genetic Contribution to Non-alcoholic Fatty Liver Disease and Prognostic Implications. Curr Diab Rep. 2021;21(3):8. 
14. VanWagner LB, Wilcox JE, Ning H, Lewis CE, Carr JJ, Rinella ME, et al. Longitudinal Association of Non-Alcoholic Fatty Liver Disease With Changes in Myocardial Structure and Function: The CARDIA Study. J Am Heart Assoc. 2020;9(4):e014279.

15. Pacifico L, Chiesa C, Anania C, De Merulis A, Osborn JF, Romaggioli S, et al. Nonalcoholic fatty liver disease and the heart in children and adolescents. World J Gastroenterol. 2014;20(27):9055-71.

16. Hallsworth K, Hollingsworth KG, Thoma C, Jakovljevic D, Macgowan GA, Anstee QM, et al. Cardiac structure and function are altered in adults with non-alcoholic fatty liver disease. J Hepatol. 2013;58(4):757-62.

17. Pacifico L, Di Martino M, De Merulis A, Bezzi M, Osborn JF, Catalano C, et al. Left ventricular dysfunction in obese children and adolescents with nonalcoholic fatty liver disease. Hepatology. 2014;59(2):461-70.

18. Bonci E, Chiesa C, Versacci P, Anania C, Silvestri L, Pacifico L. Association of nonalcoholic fatty liver disease with subclinical cardiovascular changes: A systematic review and meta-analysis. Biomed Res Int. 2015;2015:213737.

19. Treeprasertsuk S, Björnsson E, Enders F, Suwanwalaikorn S, Lindor KD. NAFLD fibrosis score: A prognostic predictor for mortality and liver complications among NAFLD patients. World J Gastroenterol. 2013;19(8):1219-29.

20. Kim D, Kim WR, Kim HJ, Therneau TM. Association between noninvasive fibrosis markers and mortality among adults with nonalcoholic fatty liver disease in the United States. Hepatology. 2013;57(4):1357-65.

21. Stepanova M, Younossi ZM. Independent Association Between Nonalcoholic Fatty Liver Disease and Cardiovascular Disease in the US Population. Clin Gastroenterol Hepatol. 2012;10(6):646-50.

22. Vancells Lujan P, Viñas Esmel E, Sacanella Meseguer E. Overview of non-alcoholic fatty liver disease (Nafld) and the role of sugary food consumption and other dietary components in its development. Nutrients. 2021;13(5):1442.

23. Purnomo HD, Mundhofir FEP, Kasno, Sudijanto E, Darmono, Daldiyono, et al. Combination of Aspartate Aminotranferase and Tumor Necrosis Factor- $\alpha$ as Non Invasive Diagnostic Tools for Non Alcoholic Steatohepatitis (NASH). Acta Med Indones. 2015;47(1):16-23.

24. Chalasani N, Younossi Z LJ. Diagnosis and Management of Nonalcoholic Fatty Liver Disease : Practice Guidelines by AASLD, ACG, AGA. Am J Gastroenterol. 2012;55:2005-23.

25. Makker J, Tariq H, Bella JN, Kumar K, Chime C, Patel $\mathrm{H}$, et al. Preclinical cardiac disease in nonalcoholic fatty liver disease with and without metabolic syndrome. Am J Cardiovasc Dis [Internet]. 2019;9(5):65-77. Available from: http://www.ncbi.nlm.nih.gov/pubmed/31763058\%0 Ahttp://www.pubmedcentral.nih.gov/articlerender.f cgi?artid=PMC6872463
26. Team R, LaBrecque DR, Abbas Z, Anania F, Ferenci $\mathrm{P}$, Khan AG, et al. World Gastroenterology Organisation global guidelines: Nonalcoholic fatty liver disease and nonalcoholic steatohepatitis. J Clin Gastroenterol. 2014;48(6):467-73.

27. Leite NC, Villela-Nogueira CA, Salles GFC, Pannain VL, Caroli-Bottino A, Cardoso CRL. Prevalence of histopathological diagnosis of steatohepatitis and fibrosis in Type 2 diabetes and interobserver agreement using Kleiner classification. Hepatology. 2009;

28. Noureddin M, Loomba R, Sc MH. Nonalcoholic Fatty Liver Disease : Indications for Liver Biopsy and Noninvasive Biomarkers. Clin Liver Dis. 2012;1(4):104-7.

29. Thong VD, Thi B, Quynh H. Correlation of Serum Transaminase Levels with Liver Fibrosis Assessed by Transient Elastography in Vietnamese Patients with Nonalcoholic Fatty Liver Disease. Int J Gen Med. 2021;14:1349-55.

30. Dowman JK, Tomlinson JW, Newsome PN. Pathogenesis of non-alcoholic fatty liver disease. QJM. 2010;103(2):71-83.

31. Hashizume H, Sato K, Yamazaki Y, Horiguchi N, Kakizaki S, Mori M. A prospective study of longterm outcomes in female patients with nonalcoholic steatohepatitis using age- and body mass indexmatched cohorts. Acta Med Okayama. 2013;67(1):45-53.

32. Miller A, McNamara J, Hummel SL, Konerman MC, Tincopa MA. Prevalence and staging of nonalcoholic fatty liver disease among patients with heart failure with preserved ejection fraction. Sci Rep [Internet]. 2020;10(1):1-9. Available from: https://doi.org/10.1038/s41598-020-69013-y

33. Bhatia LS, Curzen NP, Calder PC, Byrne CD. Nonalcoholic fatty liver disease: A new and important cardiovascular risk factor? Eur Heart J. 2012;33(10):1190-200.

34. Targher G, Valbusa F, Bonapace S, Bertolini L, Zenari L, Rodella S, et al. Non-Alcoholic Fatty Liver Disease Is Associated with an Increased Incidence of Atrial Fibrillation in Patients with Type 2 Diabetes. PLoS One. 2013;8(2):e57183.

35. Singh GK, Vitola BE, Holland MR, Sekarski T, Patterson BW, Magkos F, et al. Alterations in ventricular structure and function in obese adolescents with nonalcoholic fatty liver disease. J Pediatr. 2013;162(6):1160-1168.e1.

36. Lee C, Li H, Tsoi M, Cheung C. Association between the liver fat score ( LFS ) and cardiovascular diseases in the national health and nutrition examination survey 1999 - 2016. Ann Med [Internet]. 2021;53(1):1065-73. Available from: https://doi.org/10.1080/07853890.2021.1943514

37. Chiu LS, Pedley A, Massaro JM, Benjamin EJ, Mitchell GF, McManus DD, et al. The association of non-alcoholic fatty liver disease and cardiac structure and function-Framingham Heart Study. Liver Int. 2020;40(10):2445-54.

38. Lee H, Kim G, Lee YH. Association between nonalcoholic steatohepatitis and left ventricular diastolic dysfunction in type 2 diabetes mellitus. Diabetes Metab J. 2020;44(3):486-7. 
39. Hu KC, Wang HY, Liu SC, Liu CC, Hung CL, Bair MJ, et al. Nonalcoholic fatty liver disease: Updates in noninvasive diagnosis and correlation with cardiovascular disease. World J Gastroenterol. 2014;20(24):7718-29.
40. Liu H, Cao Y, Jin J, Hua Q, Li Y, Guo Y. Liver Fibrosis Scoring Systems as Novel Tools for Predicting Cardiovascular Outcomes in Patients Following Elective. J Am Heart Assoc. 2021;10(2):e018869. 